\title{
HIGHLIGHTS
}

ATRIAL FIBRILLATION

\section{HAS-BLED-a new risk score to predict bleeding in patients with AF}

A risk score derived and validated by an international team of researchers has identified heart failure and diabetes mellitus as novel risk factors for bleeding in patients with atrial fibrillation (AF) who are receiving anticoagulant therapy. Moreover, this model-the HAS-BLED (The Birmingham AF Bleeding Schema: Hypertension, Abnormal Renal/Liver Function, Stroke, Bleeding History or Predisposition, Labile INR, Elderly, Drugs/ Alcohol Concomitantly) score-provided the most-accurate prediction of bleeding risk in a comparison with four other risk scores.

\section{4 ...the predictive value of HAS- BLED ... was the most accurate of the five models tested 77}

Treatment with anticoagulant medication is an effective means of preventing stroke and thromboembolism in patients with AF. However, such therapy places these individuals at increased risk of bleeding events. Some patients experience bleeding more than others, but predicting who is most at risk is not an easy task. "There is an unmet need for a simple, user-friendly score to assess bleeding risk in AF patients in everyday clinical practice," explains
Professor Gregory Lip from Birmingham, UK, who was one of the investigators. The existing risk scores have several limitations; most were derived from cohorts of patients receiving anticoagulant therapy who did not have AF, and some employ very complex scoring systems that make their daily use impractical for the busy physician.

The HAS-BLED score was initially derived in a group of patients with AF from the Euro Heart Survey. The latest report from Lip and colleagues builds on this earlier work to validate the score in 7,329 participants of the SPORTIF III and V trials, in which warfarin and ximelagatran for stroke prevention were compared in the setting of AF.

Patients in these study populations were generally elderly (mean age 72 years), $77 \%$ had hypertension, and around half had coronary artery disease. The mean $\mathrm{CHADS}_{2}$ score for stroke risk was 2.4. Bleeding events were experienced by 217 (3\%) patients. Multivariate analysis indicated that age $\geq 75$ years, diabetes, left ventricular dysfunction, renal impairment, and concurrent use of aspirin were significant predictors of bleeding.

The predictive value of HAS-BLED for bleeding was the most accurate of the five models tested (c-statistic 0.66, compared with $0.52-0.63$ for the other four models),

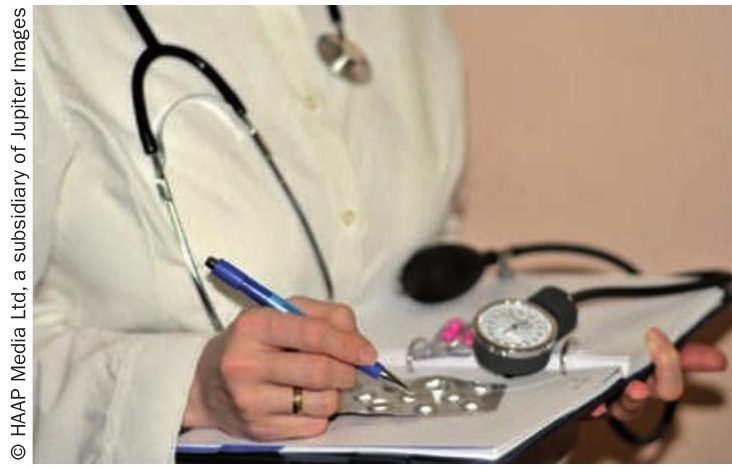

with the number of bleeding events increasing steadily with increasing HASBLED score. Using this model, 20.4\% of patients were classed as being at low risk of bleeding, and these individuals had a bleeding rate of $<1 \%$ per year. This rate was almost twofold higher among patients classified as low risk in the other models.

Looking to the future, the HAS-BLED score has "the possibility of deciding on dose of new oral anticoagulant agents that could be an alternative to warfarin," reveals Professor Lip. He cautions, however, that the score "would need to be evaluated in a 'real-world' patient cohort, and this work is in progress".

Alexandra King

Original article Lip, G. Y. et al. Comparative validation of a novel risk score for predicting bleeding risk in anticoagulated patients with atrial fibrillation: the HAS-BLED Score. J. Am. Coll. Cardiol. doi:10.1016/j. jacc.2010.09.024 